\title{
Mutual Clutter Suppression Techniques for FM Sonars
}

\author{
Jacek MARSZAL, Mariusz RUDNICKI, Andrzej JEDEL, Roman SALAMON, Iwona KOCHAŃSKA \\ Department of Marine Electronic Systems \\ Faculty of Electronics, Telecommunications and Informatics \\ Gdńsk University of Technology \\ Narutowicza 11/12, 80-233 Gdańsk, Poland; e-mail: jacek.marszal@eti.pg.gda.pl
}

(received April 18, 2016; accepted August 4, 2016)

\begin{abstract}
The article presents methods that help in the elimination of mutual clutter as well as the consequences of two FM sounding signal sonars operating in the same body of water and frequency band. An in-depth analysis of mutual clutter was carried out. The effects of sounding signal differentiation were determined, as was the Doppler effect on mutual clutter suppression. One of the methods analysed is of particular interest in a situation in which collaborating sonars are operating in opposite frequency modulation directions. This method is effective for both linear and hyperbolic frequency modulations. A formula was derived, identifying exactly how much quantities of clutter may be lessened. The work included comprehensive computer simulations and measurements as well as tests in real-life conditions.
\end{abstract}

Keywords: sonar; signal processing; FM; LFM; HFM; clutter; crosstalk.

\section{Introduction}

The search for underwater targets during military operations at sea is usually conducted by a group of ships or helicopters, each of which uses identical sonars. As well as receiving echo signals, the sonars receive the sounding signals of other sonars operating nearby. Due to the fact that transmission loss in communications systems is only one way, the maximum distance between neighbouring sonars producing substantial interference is much greater than the nominal ranges of the sonars (Marszal, Salamon, 2014; Salamon, 2006). With no synchronization of sounding signal emissions within sonar groups, interference occurs at random, but tends to appear at specific periods. While system operators are usually able to be distinguished from echo signals, they make detection unnecessarily more problematic. The majority of classic sonars with narrow frequency sounding signals are in a position to choose between one of several operating frequencies, which usually solves the problem of mutual clutter entirely. Where broadband sounding signal sonars are concerned, however, the limited bandwidth of transducers makes the selection of the operating band a technical impossibility. In such cases, mutual clutter can be resolved by employing a special search tactic that takes account of sonar array beam patterns and the distances between sonars. The idea of using frequency modulation sounding signals that have a varying (either ascending or descending) direction of modulation, was presented by the authors in a previous work (JEDEL et al., 2001). The objective of this article is to offer an in-depth theoretical analysis and an experimental verification of mutual clutter suppression involving two FM sounding signal sonars operating in the same frequency band. The subject of mutual clutter within sonar systems is also covered in the works of other authors, e.g. (ElMINOWICZ, ZAJĄCZKOWSKI, 2007; Meller, 2014; PJachev, 2008; Ricks, 1994).

\section{Methods of sonar mutual crosstalk suppression}

The subject of the analysis herein is sonar emitting broadband sounding pulses with a linear (LFM) or hyperbolic (HFM) frequency modulation (YANG, SARKAR, 2006). The LFM signal can be described by the following formula:

$$
s(t)=s_{o} \exp \left[j 2 \pi\left(f_{o}-\frac{B}{2}+\frac{B}{2} \frac{t}{T}\right) t\right] \quad 0<t<T,
$$

where $t[\mathrm{~s}]$ - time, $f_{o}[\mathrm{~Hz}]$ - carrier frequency, $B[\mathrm{~Hz}]$ - signal bandwidth, $T[\mathrm{~s}]$ - duration of the sounding pulse. If bandwidth $B=f_{h}-f_{l}\left(f_{l}-\right.$ lower, and $f_{h}-$ 
upper spectrum frequency), then the signal frequency increases over time. A change in the character of $B$ results in frequency decreasing with time.

The HFM signal is written as follows:

$$
s_{h}(t)=s_{o} \exp \left[j 2 \pi a \ln \left(1+b \frac{t}{T}\right)\right] \quad 0<t<T,
$$

where $b=\left(f_{l}-f_{h}\right) / f_{h}, a=T f_{l} / b$. For this signal notation, frequency increases with time. Frequency decreases with time when $b=\left(f_{h}-f_{l}\right) / f_{l}, a=T f_{h} / b$ is inserted.

Figure 1 shows the amplitude spectra $|S(f)|$ of both signals, and Fig. 2 shows how their frequencies change in the function of time. As frequencies change up and down, the amplitude spectra become identical. Whether the frequency goes up or down is known from the phase spectra. Figure 2 shows a derivative of the phase spectrum, which, as we know, is the instantaneous signal frequency. These, as well as the sub-

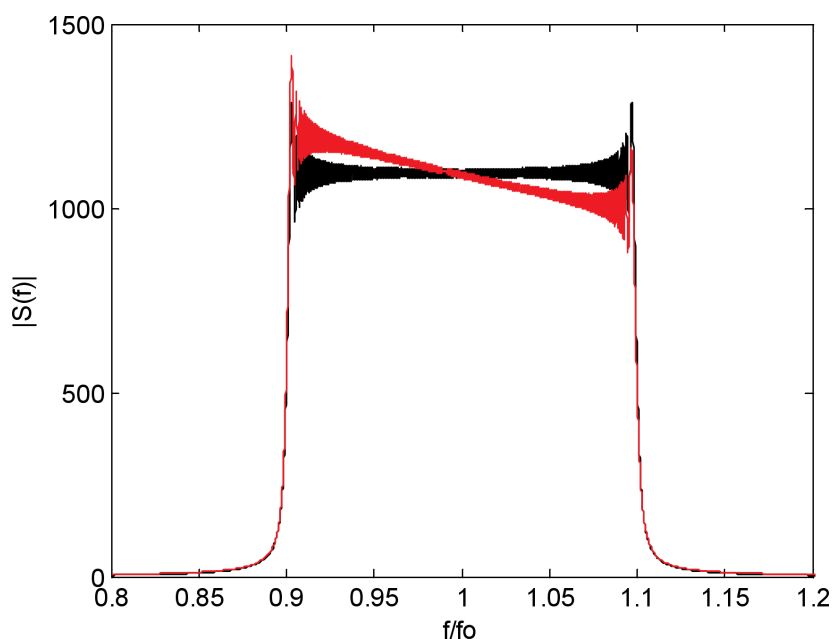

Fig. 1. Amplitude spectra of LFM (black line) and HFM signals (red line), $T=1 \mathrm{~s}, B=3 \mathrm{kHz}$.

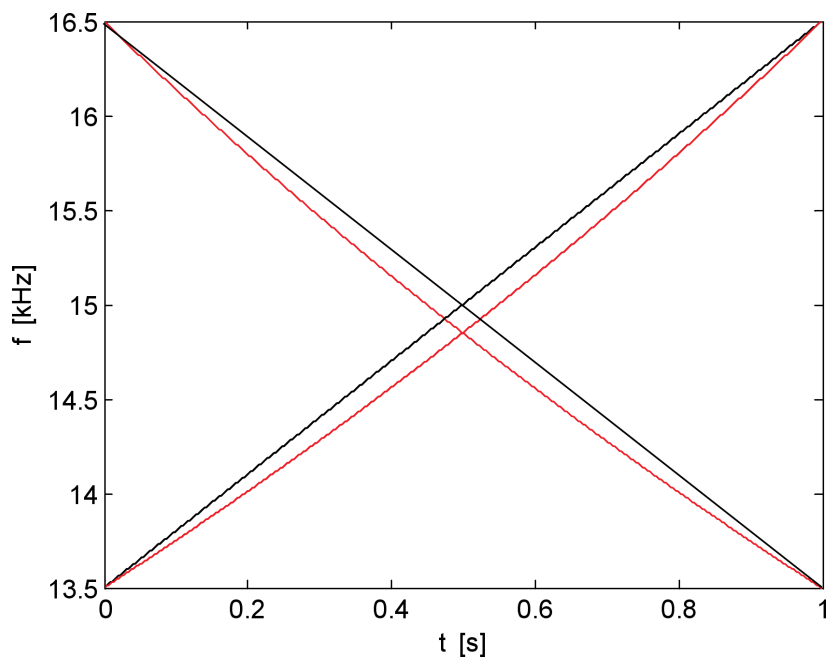

Fig. 2. Change in LFM (black line) and HFM (red line) signal frequency $T=1 \mathrm{~s}, B=3 \mathrm{kHz}$. sequent results of calculations, were carried out for $f_{o}=15 \mathrm{kHz}, s_{o}=1$.

The sonar receivers perform matched filtration. Once detected, the signal is described by the following formula (SALAMON, 2006):

$$
y(t)=\left|\mathcal{F}^{-1}\left\{X(f) S^{*}(f)\right\}\right|,
$$

where $X(f)$ is the Fourier transform of the signal being received, and $S(f)$ - the Fourier transform of the LFM or HFM sounding signal. The signal received is either a useful echo signal or a sounding signal from the sonar nearby (clutter). Assuming that the signals have an identical amplitude will help us directly determine how well the unmatched crosstalk signal might reduce output signal amplitude $y(t)$.

Let us also assume that echo signals are the copies of LFM and HFM sounding signals which are delayed by time $t_{o}$. In this case, the plots of signals at the matched filter output are as presented in Fig. 3, mak-
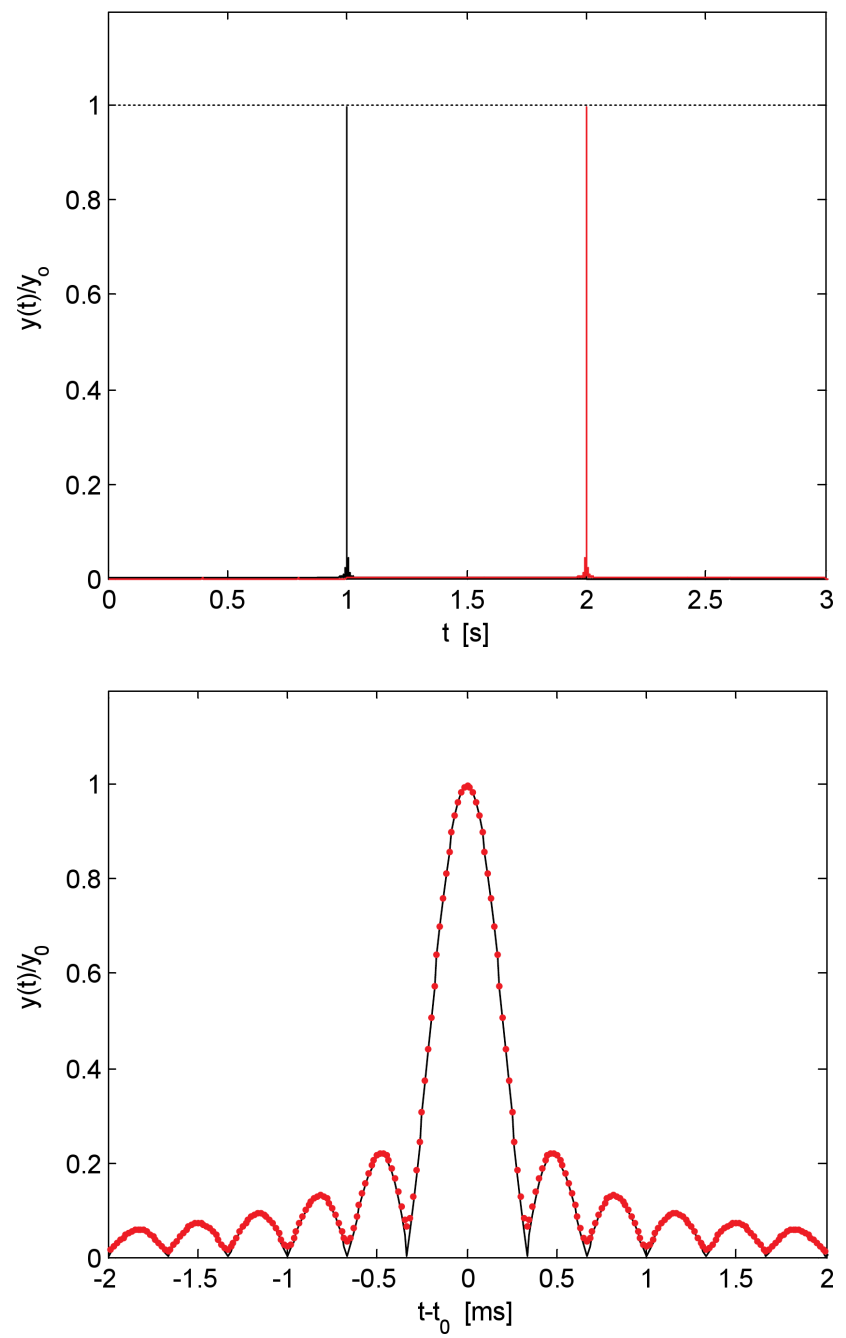

Fig. 3. Echo signals at matched receiver output for LFM (black line) and HFM signals (red line), $B=3 \mathrm{kHz}, T=$ $1 \mathrm{~s}, t_{o}=1 \mathrm{~s}, y_{o}=6 \cdot 10^{4}$, the scale of the bottom chart has been enlarged. 
ing them practically indiscernible from these two types of modulation. Their maximum value $y_{o}$ is proportional to the energy of the echo signal, and amounts to $y_{o}=s_{o}^{2} T f_{s}$ for numerical calculations. As we know (SALAMON, 2006), this has no relation to the signal waveform (its spectrum), which is the reason for its being identical for both the LFM and HFM signals.

If the adjacent sonars emit identical sounding signals and their receivers perform matched filtration, then the crosstalk signal size will depend on how far apart the sonars and the angles at which they are positioned for transmitting and receiving beam patterns are. Since these are random parameters, this paper is not going to deal with them. Instead, focus more on the effect an unmatched sounding signal of one sonar has on another sonar's receiver filter. The signal crosstalk suppression $C S$ will be equal to the quotient's logarithmic value:

$$
C S=20 \log \frac{y_{p}}{y_{0}},
$$

where $y_{p}$ is the maximum clutter signal value at the output of the filter matched to the sounding signal.

It is clear that $C S=0$, when the sounding signals of both sonars are identical $\left(y_{0}=y_{p}\right)$. The desired $C S<0$ values will appear if there is a difference between the sounding signals of both sonars; that is to say that the filter characteristics will be mismatched with the crosstalk signal. This mismatch may be brought about by differences in:

- the type of pulse (LFM or HFM),

- the direction of frequency change (chirp up or chirp down) for same pulse type,

- the direction of frequency change (chirp up or chirp down) for different pulse types,

- frequency band location,

- bandwidth,

- pulse duration.

The first above three differences may be introduced as permanent settings in the group of sonars and they ensure that the mutual clutter will be reduced to the levels shown further. They have no impact on sonar operating parameters and cause no deterioration. Signal spectra overlap can be avoided if the spectra are adequately spaced along the frequency axis. In practice, the bandwidth must be halved to guarantee that the spectra may fit into the transmitter and receiver carrier bands. While this procedure is successful in eliminating crosstalk, it does however deteriorate sonar quality (reducing signal to noise ratio and deteriorating range resolution). The other differences, not listed above, are the results of new settings made while the sonars are in operation and cannot therefore be implemented as a means of suppressing crosstalk.

Figure 4 shows the runs of signals at the output of the filter matched to the LFM signal, when an HFM

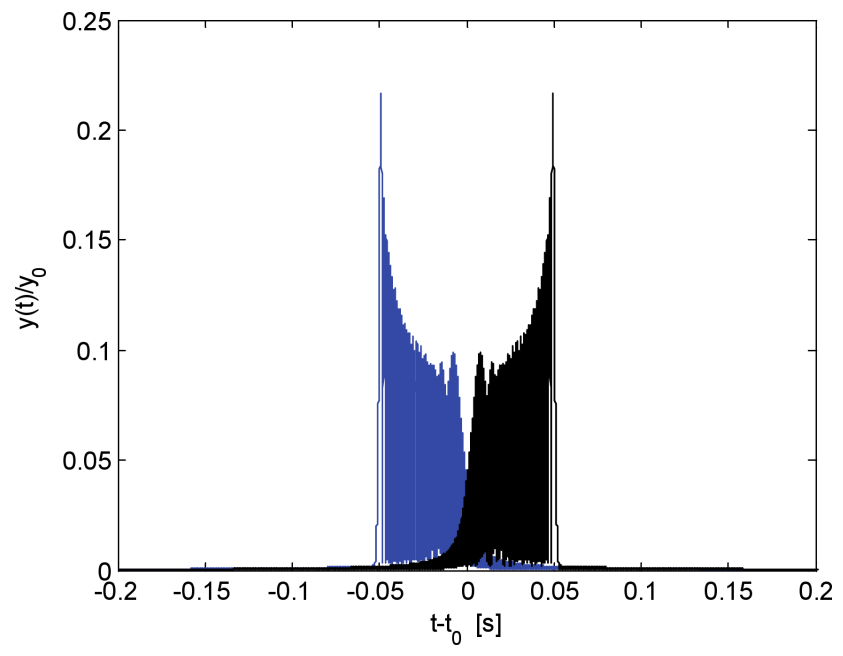

Fig. 4. HFM crosstalk signals at LFM filter output for the same direction of frequency change, (black curve - ascending frequency, blue curve - descending frequency), $T=1 \mathrm{~s}$,

$$
B=3 \mathrm{kHz} \text {. }
$$

signal appears at the input. Changes in frequency directions are consistent. As can be seen here, crosstalk suppression is low, and amounts to $C S=-13.3 \mathrm{~dB}$, rendering this method of crosstalk suppression ineffective. Figure 5 illustrates the effect of frequency direction change on LFM signals. The sounding signal frequency increases over time, whereas that of the crosstalk signal decreases. When reversed, the results are identical. Crosstalk suppression is significant and amounts to $C S=-35.2 \mathrm{~dB}$.

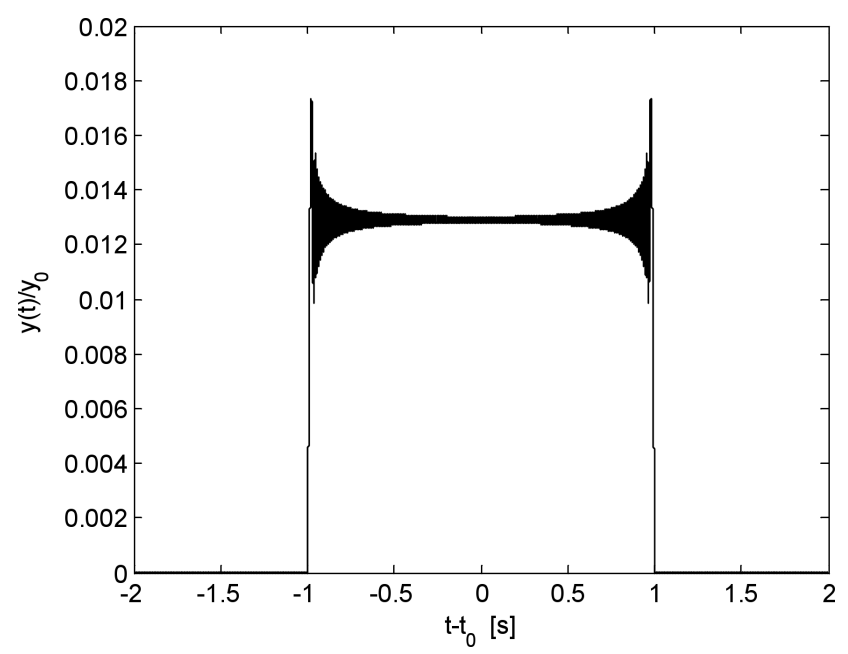

Fig. 5. LFM crosstalk signals at LFM filter output for opposite directions of frequency change, $T=1 \mathrm{~s}, B=3 \mathrm{kHz}$.

A similar level of crosstalk suppression is achieved for HFM signals, shown in Fig. 6, which amounts itself to $C S=-34.4 \mathrm{~dB}$. The shape of the signal depends on the direction of frequency changes in the matched filter. 


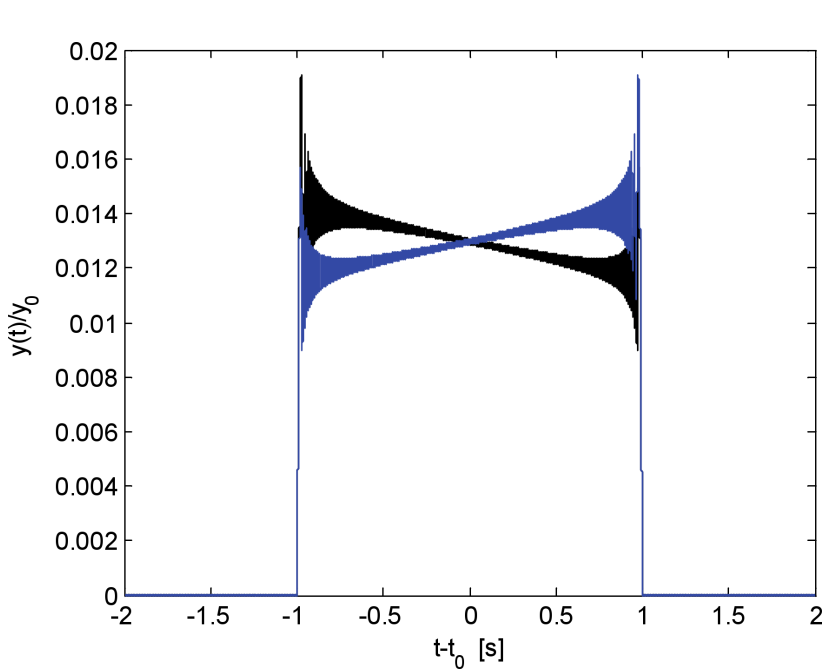

Fig. 6. HFM crosstalk signals at HFM filter output for opposite directions of frequency change (black curve - ascending for the signal, descending for filtration, blue curve - the other way round).

If one of the signals is of the LFM type and the other is of the HFM type, and the directions of frequency change over time go in opposite directions, then the signals at the filter output will be as presented in Fig. 7. Here, crosstalk suppression is similar and is $C S=-35.0 \mathrm{~dB}$.

As well as supressing the crosstalk signal level, a failure in matching increases duration of the crosstalk signal. As a result of this, crosstalk signals are easier to distinguish from significantly shorter useful echo signals. In the examples shown in the Figures, the duration of an echo pulse (after matched filtration) from point target is $\tau=1 / B=0.33 \mathrm{~ms}$, and the crosstalk pulse duration is equal to $2 \mathrm{~T}$ (in the presented example $2 \mathrm{~s}$ ). This is how long the function of correlation between the sounding signal and crosstalk signal is, as the filtration described with formula (3) is the method in which correlation is performed in the frequency domain.

The values of crosstalk suppression depend on pulse duration $T$ as well as the width of their spectrum $B$. These values are given in Table 1 , and are calculated assuming that they are identical for both echo

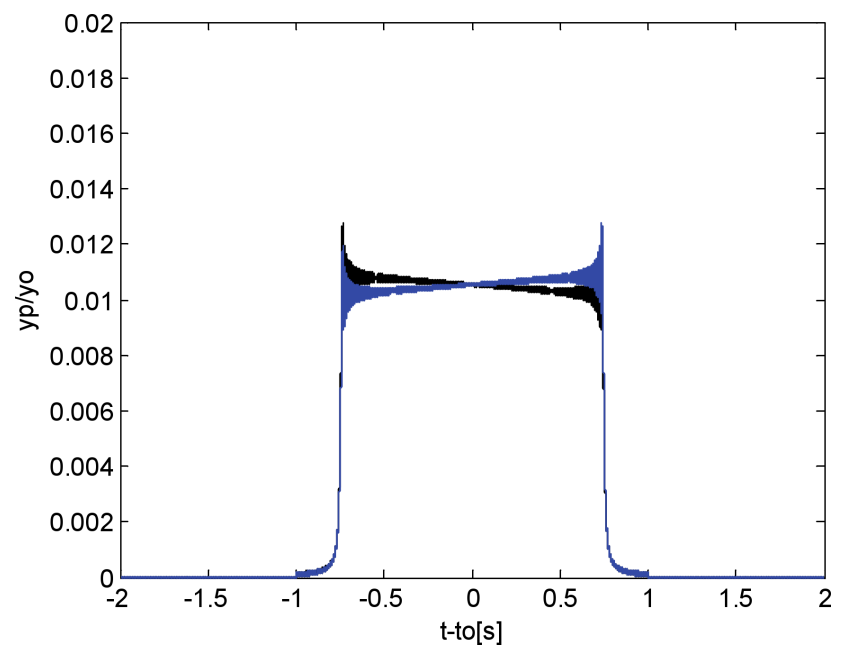

Fig. 7. LFM crosstalk signals at HFM filter output for opposite directions of frequency change (black curve - ascending for the signal, descending for filtration, blue curve - the other way round).

and crosstalk signals. The changes in sounding signal and crosstalk signal frequencies go in opposite directions. These computations have shown that the carrier frequency has no real effect on crosstalk suppression.

As the numerical computations in Table 1 show, the greater the $B$ bandwidth and the $T$ pulse duration are, the greater crosstalk suppression is. This relation can be illustrated with the following approximate formula, whose error does not exceed $0.5 \mathrm{~dB}$ :

$$
C S \cong-10 \log (B T)[\mathrm{dB}] .
$$

Additional crosstalk suppression may be the result of differing durations of sounding and crosstalk signals. Figure 8 shows examples of crosstalk signal change when the filter matches a pulse with $T$ duration and the duration of crosstalk pulse is $T_{p}$. The magnitude of the crosstalk signal is normalised relative to the echo signal at the output of the filter that matches the signal. The direction of the sounding signal and crosstalk signal frequency change is the same, and bandwidth of the spectrum of both signals is $B=3 \mathrm{kHz}$.

Table 1. Crosstalk suppression for frequency changes in opposite directions.

\begin{tabular}{|c|c|c|c|c|c|c|c|c|c|c|c|}
\hline \multirow{3}{*}{\multicolumn{2}{|c|}{$C S[\mathrm{~dB}]$}} & \multirow{2}{*}{\multicolumn{5}{|c|}{$\begin{array}{c}\text { LFM } \\
T[\mathrm{~ms}] \\
\end{array}$}} & \multirow{2}{*}{\multicolumn{5}{|c|}{$\begin{array}{l}\text { HFM } \\
T[\mathrm{~ms}] \\
\end{array}$}} \\
\hline & & & & & & & & & & & \\
\hline & & \multirow{2}{*}{$\begin{array}{c}50 \\
-11.4 \\
\end{array}$} & \multirow{2}{*}{$\begin{array}{c}125 \\
-15.4\end{array}$} & \multirow{2}{*}{$\begin{array}{c}250 \\
-18.4\end{array}$} & \multirow{2}{*}{$\begin{array}{c}500 \\
-21.4 \\
\end{array}$} & \multirow{2}{*}{$\begin{array}{c}1000 \\
\mathbf{- 2 4 . 4}\end{array}$} & \multirow{2}{*}{$\begin{array}{c}50 \\
-11.4\end{array}$} & \multirow{2}{*}{$\begin{array}{c}125 \\
-15.3\end{array}$} & \multirow{2}{*}{\begin{tabular}{|c|}
250 \\
-18.4 \\
\end{tabular}} & \multirow{2}{*}{$\begin{array}{c}500 \\
-21.4 \\
\end{array}$} & \multirow{2}{*}{$\begin{array}{c}1000 \\
-24.4\end{array}$} \\
\hline \multirow{5}{*}{$B[\mathrm{~Hz}]$} & 250 & & & & & & & & & & \\
\hline & 500 & -14.4 & -18.4 & -21.4 & -24.4 & -27.4 & -14.3 & -18.3 & -21.3 & -24.3 & -27.3 \\
\hline & 1000 & -17.4 & -21.3 & -24.4 & -27.4 & -30.5 & -17.2 & -21.2 & -24.2 & -27.2 & -30.2 \\
\hline & 2000 & -20.5 & -24.4 & -27.4 & -30.5 & -33.5 & -19.9 & -23.9 & -26.9 & -29.9 & -32.9 \\
\hline & 3000 & -22.2 & -26.2 & -29.2 & -32.2 & -35.2 & -21.4 & -25.4 & -28.4 & -31.4 & -34.4 \\
\hline
\end{tabular}




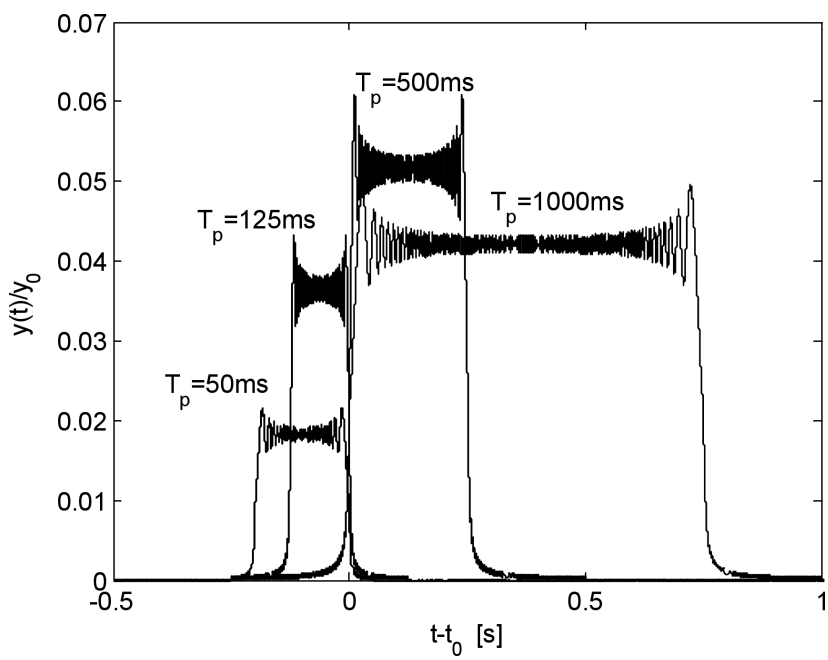

Fig. 8. LFM crosstalk signals at LFM filter output for the same direction of frequency change, $T=250 \mathrm{~ms}$, $B=3 \mathrm{kHz}$.

Figure 9 shows the crosstalk signals when the direction of frequency change is opposite, in relation to the direction of echo signal frequency change. The magnitude of the crosstalk signal is normalised relative to

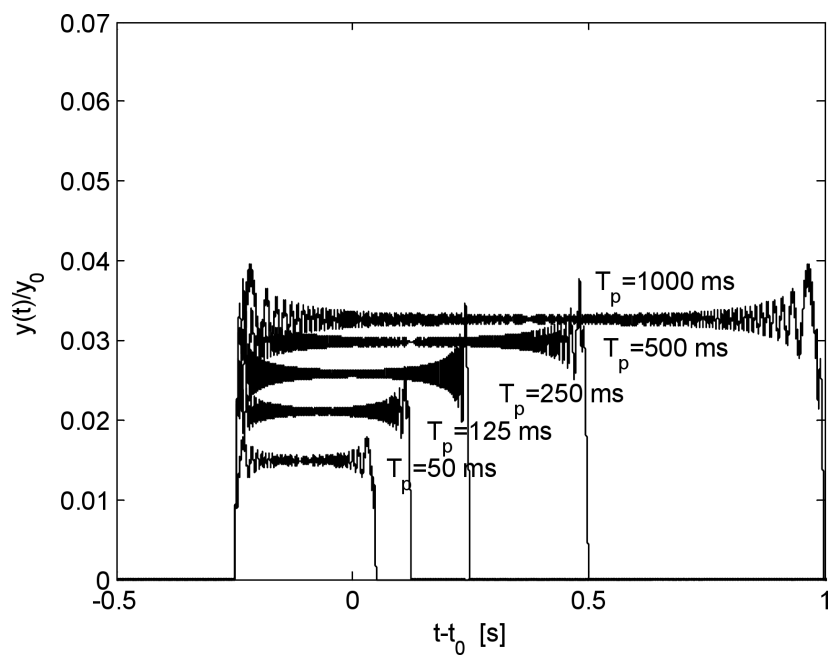

Fig. 9. LFM crosstalk signals at LFM filter output for opposite directions of frequency change, $T=250 \mathrm{~ms}$, $B=3 \mathrm{kHz}$. the echo signal at the output of the filter matching the signal. The bandwidth of the spectrum of both signals is $B=3 \mathrm{kHz}$.

In both cases shown in above figures, the change of crosstalk pulse duration reduces maximum crosstalk size significantly. When velocity change occurs in an opposing direction, suppression is stronger for longer durations, $T_{p}$. Table 2 lists the computation results for different combinations of durations $T$, and $T_{p}$ of LFM pulses, when modulation directions are the same and/or different.

As Table 2 shows, crosstalk suppression is much stronger for longer durations $T$ of the pulse with matching filter. As an example, when the directions of frequency changes are the same, for $T=1 \mathrm{~s}$ and $T_{p}=50 \mathrm{~ms}$, we have $C S=-46.1 \mathrm{~dB}$, and for $T=$ $50 \mathrm{~ms}$ and $T_{p}=1 \mathrm{~s}, C S=-20.1 \mathrm{~dB}$. In both cases, maximum crosstalk signal values at the matched filter output are identical, but for $T=1 \mathrm{~s}$, the maximum echo signal $y_{o}$ is greater by $26.0 \mathrm{~dB}$.

When echo and crosstalk signal frequency changes occur in opposite directions, crosstalk suppression tends to be slightly stronger, except in cases in which echo and crosstalk signals durations are the same. When the directions of frequency changes are the same, suppression does not occur as the filter is matched to both signals. Crosstalk suppression only occurs for opposite directions of frequency change. This is represented by the bold type in Tables 1 and 2, which demonstrate how strong crosstalk suppression is; the values being compared herein feature in Table 1 , in the last row.

Figures 10 and 11 show the effect of mismatched filter and crosstalk signal characteristics on the crosstalk suppression caused by differences in bandwidth. The first figure illustrates LFM crosstalk signals that have the same direction of frequency change when filter bandwidth is equal to $B=3 \mathrm{kHz}$. In the following figure with the same bandwidth $B$, the frequency changes in the opposite direction. The spectrum bandwidth of the crosstalk signal is marked as $B_{p}$.

When the directions of frequency change are identical, crosstalk suppression is stronger for greater differences between bandwidth $B$ and the bandwidth of

Table 2. Crosstalk suppression as a result of different pulse duration $(B=3 \mathrm{kHz})$.

\begin{tabular}{|c|c|c|c|c|c|c|c|c|c|c|c|}
\hline \multirow{4}{*}{\multicolumn{2}{|c|}{$C S[\mathrm{~dB}]$}} & \multicolumn{10}{|c|}{ Direction of frequency change } \\
\hline & & \multirow{2}{*}{\multicolumn{5}{|c|}{$\begin{array}{l}\text { same } \\
T[\mathrm{~ms}]\end{array}$}} & \multirow{2}{*}{\multicolumn{5}{|c|}{$\begin{array}{c}\text { opposite } \\
T[\mathrm{~ms}]\end{array}$}} \\
\hline & & & & & & & & & & & \\
\hline & & 50 & 125 & 250 & 500 & 1000 & 50 & 125 & 250 & 500 & 1000 \\
\hline \multirow{5}{*}{$T_{p}[\mathrm{~ms}]$} & 50 & 0 & -26.0 & -33.3 & -39.9 & -46.1 & -22.2 & -29.3 & -34.9 & -40.7 & -46.5 \\
\hline & 125 & -18.0 & 0 & -27.3 & -35.1 & -41.8 & -21.3 & -26.2 & -31.5 & -37.1 & -42.8 \\
\hline & 250 & -19.3 & -21.3 & 0 & -30.3 & -38.1 & -20.9 & -25.5 & -29.2 & -34.5 & -40.1 \\
\hline & 500 & -19.9 & -23.1 & -24.3 & 0 & -33.4 & -20.7 & -25.0 & -28.5 & -32.2 & -37.5 \\
\hline & 1000 & -20.1 & -23.7 & -26.1 & -27.3 & 0 & -20.5 & -24.7 & -28.0 & -31.5 & -35.2 \\
\hline
\end{tabular}




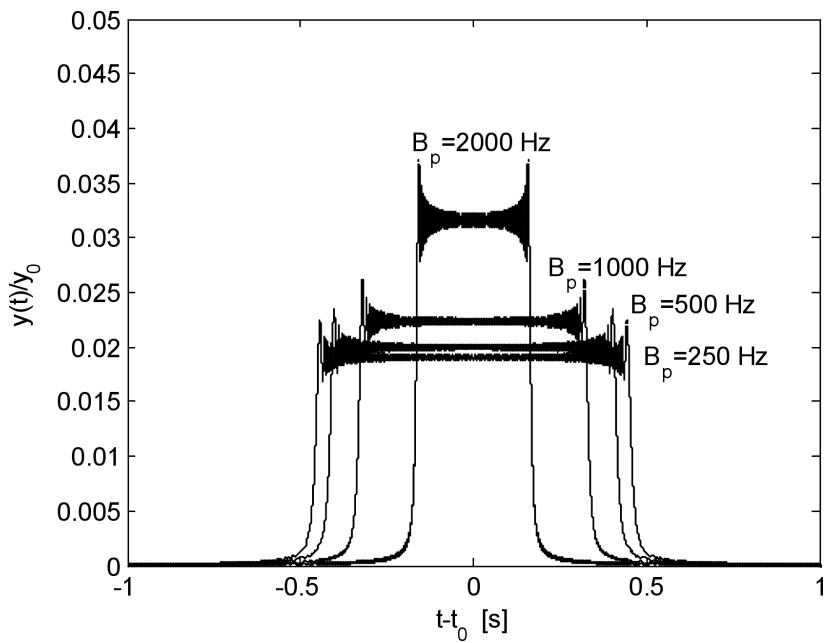

Fig. 10. LFM crosstalk signals at LFM filter output for identical direction of frequency change, $T=1 \mathrm{~s}$, $B=3 \mathrm{kHz}$.

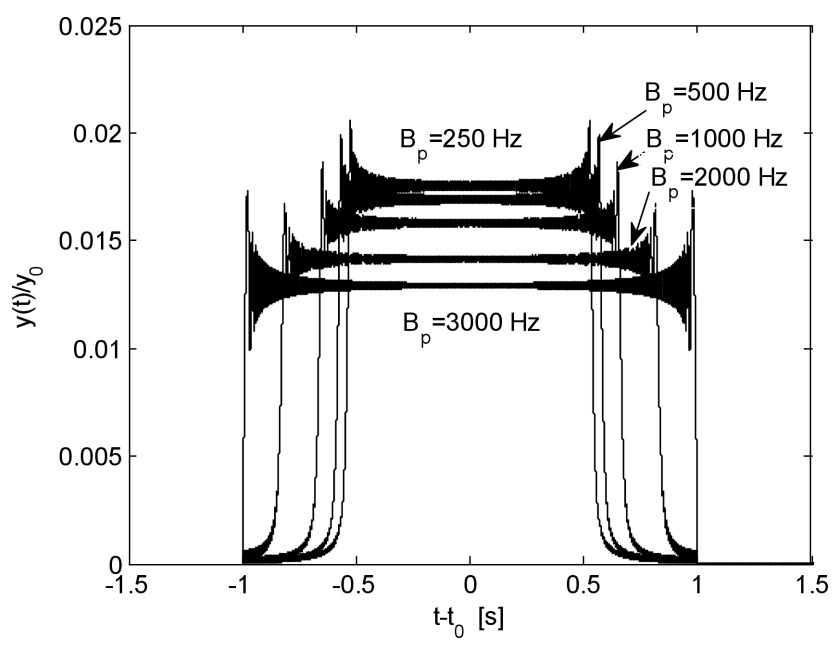

Fig. 11. LFM crosstalk signals at LFM filter output for opposite direction of frequency change, $T=1 \mathrm{~s}$,

$$
B=3 \mathrm{kHz} \text {. }
$$

crosstalk signal spectrum $B_{p}$. The reverse is the case when frequencies change in opposing directions. Crosstalk suppression is even stronger in such cases. This tendency can be seen in Table 3, which shows the results for all combinations of bandwidth of echo and crosstalk signals.
Crosstalk values in bold type refer to the situation when the bandwidth of both echo and crosstalk signals are the same. These can also be found in the last column of Table 1 . When we compare the results shown in Tables 2 and 3, we can see that crosstalk suppression is enhanced to an even greater degree. This is the result of different echo pulse and crosstalk durations and the fact that their bandwidths do not match.

\section{Crosstalk suppression in the presence of the Doppler effect}

The Doppler effect occurs in sonar as it moves in relation to its target. This may diminish detection and produce errors in target distance determination. Detection conditions deteriorate when the sonar emits ping type and LFM signals. This adverse effect can be eliminated through the use of HFM signals. While there are no errors in determining distance to target for ping signals, for LFM and HFM signals errors are identical, and increase for longer durations of the sounding pulse. We will demonstrate further how the Doppler effect affects the magnitude of the crosstalk signal when sonars move in relation to each other with an axial velocity $v$. Axial velocity is a component of velocity projected on a straight line linking sonars. We will evaluate how the Doppler effect affects crosstalk magnitude as before, by assuming identical amplitudes of echo signal and crosstalk signal.

We will note down signals with the Doppler effect as being the effect of time compression (YANG, SARKAR, 2006), that is to say for signal LFM, from the transformed formula 1, as:

$$
s(t)=s_{o} \exp \left[j 2 \pi\left(f_{o}-\frac{B}{2}+\frac{B}{2} \frac{k t}{T}\right) k t\right] \quad 0<t<T / k
$$

and for the HFM signal, from transformed formula 2, as:

$$
s_{h}(t)=s_{o} \exp \left[j 2 \pi a \ln \left(1+b \frac{k t}{T}\right)\right] \quad 0<t<T / k
$$

where $k=1+v / c, c-$ being the velocity of the acoustic

\begin{tabular}{|c|c|c|c|c|c|c|c|c|c|c|c|}
\hline \multirow{4}{*}{\multicolumn{2}{|c|}{$C S[\mathrm{~dB}]$}} & \multicolumn{10}{|c|}{ Direction of frequency change } \\
\hline & & \multirow{2}{*}{\multicolumn{5}{|c|}{$\frac{\text { same }}{B[\mathrm{~Hz}]}$}} & \multirow{2}{*}{\multicolumn{5}{|c|}{$\begin{array}{c}\text { opposite } \\
B[\mathrm{~Hz}]\end{array}$}} \\
\hline & & & & & & & & & & & \\
\hline & & 250 & 500 & 1000 & 2000 & 3000 & 250 & 500 & 1000 & 2000 & 3000 \\
\hline \multirow{5}{*}{$B_{p}[\mathrm{~Hz}]$} & 250 & 0 & -22.5 & -27.4 & -31.0 & -33.0 & -24.4 & -27.2 & -29.6 & -32.1 & -33.7 \\
\hline & 500 & -22.5 & 0 & -25.6 & -30.4 & -32.6 & -27.2 & -27.4 & -30.3 & -32.6 & -34.0 \\
\hline & 1000 & -27.4 & -25.6 & 0 & -28.6 & -31.6 & -29.6 & -30.3 & -30.5 & -34.4 & -34.6 \\
\hline & 2000 & -31.0 & -30.4 & -28.6 & 0 & -28.6 & -32.1 & -32.6 & -33.4 & -33.5 & -34.6 \\
\hline & 3000 & -33.0 & -32.6 & -31.6 & -28.6 & 0 & -33.7 & -34.0 & -34.6 & -33.5 & -35.2 \\
\hline
\end{tabular}
wave in water.

Table 3. Crosstalk suppression as a result of changes in pulse bandwidth $(T=1 \mathrm{~s})$. 
Figures 12 and 13 show the effect of the Doppler effect, in which the settings of both of the sonars are identical, but the bandwidth of the sounding signals is changed. The figures shown confirm the above properties of the LFM and HFM signals and also illustrate how bandwidth affects the crosstalk signal. Another effect that has no bearing on the problem in question, is a shift in correlation maximum position relative to $t_{0}$. When this type of phenomenon affects the echo returned from a moving target, this results in a serious error in distance determination (MARszal, SALAMON, 2012; Marszal, 2014).

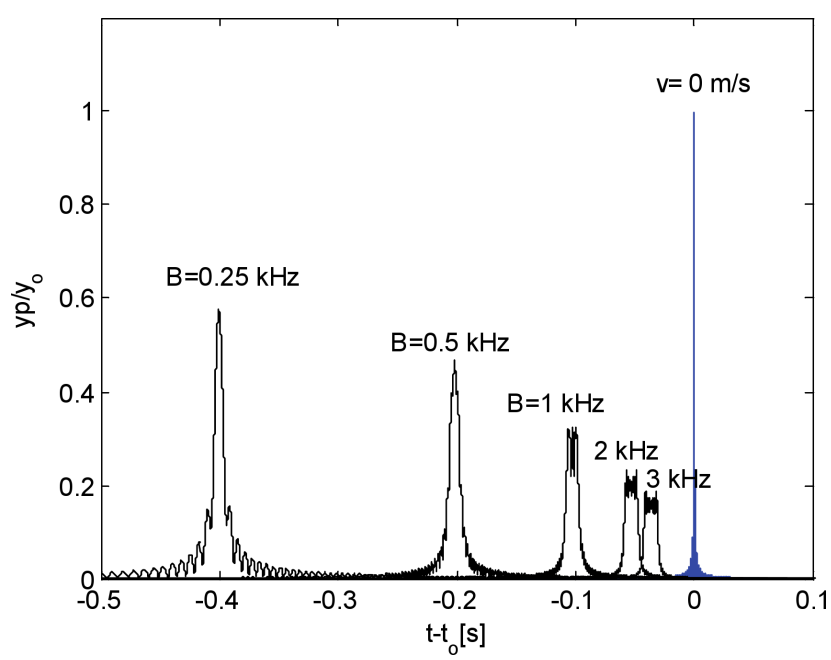

Fig. 12. LFM crosstalk signals at LFM filter output, $T=1 \mathrm{~s}, f_{0}=15 \mathrm{kHz}, v=10 \mathrm{~m} / \mathrm{s}$.

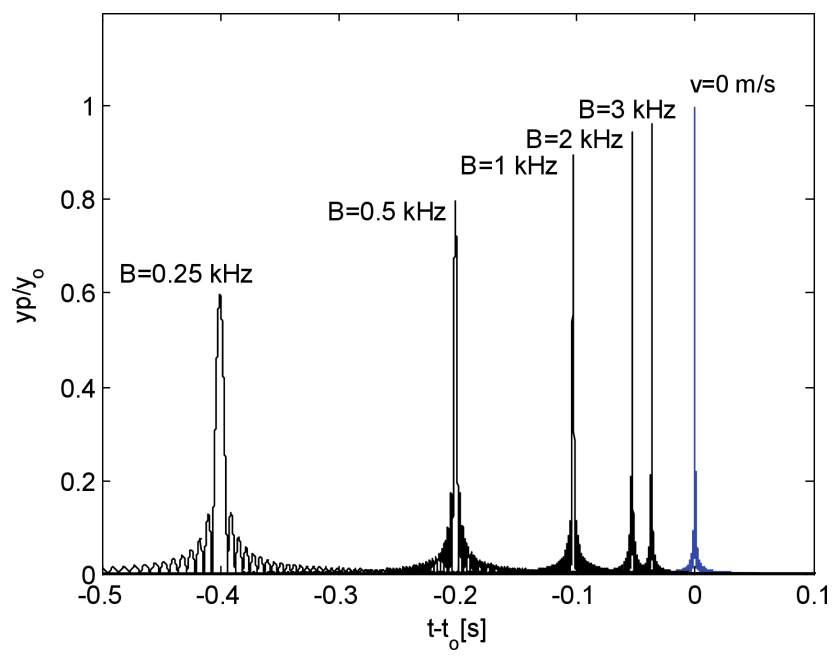

Fig. 13. HFM crosstalk signals at HFM filter output, $T=1 \mathrm{~s}, f_{0}=15 \mathrm{kHz}, v=10 \mathrm{~m} / \mathrm{s}$.

The Doppler effect's impact on crosstalk for opposite directions of frequency change, in both sonars, is shown in the consecutive figures. Figure 14 shows the LFM crosstalk signal in both the absence and presence of the Doppler effect. Figure 15 shows the analogous

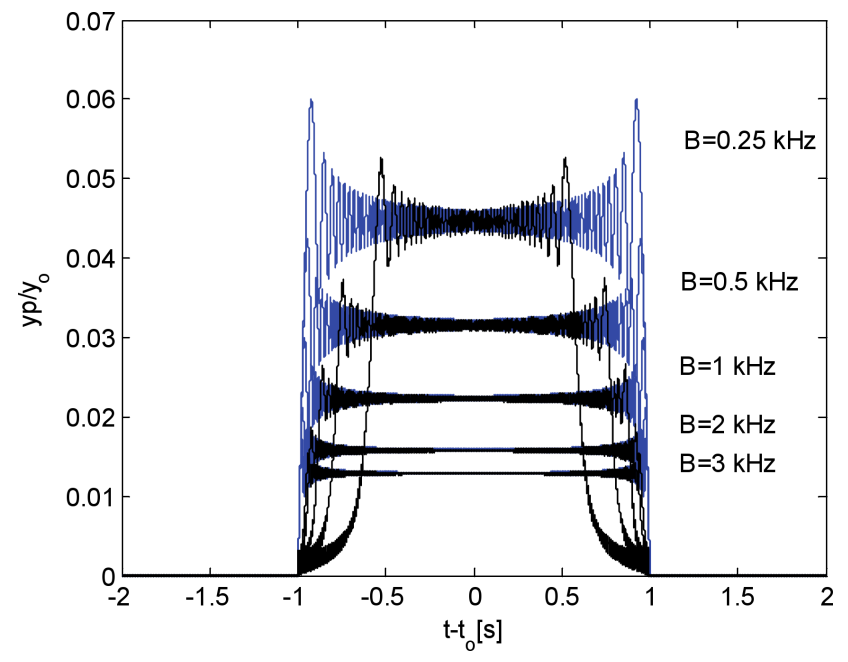

Fig. 14. LFM crosstalk signals at LFM filter output, blue line $v=0 \mathrm{~m} / \mathrm{s}$, black line, $v=10 \mathrm{~m} / \mathrm{s}, T=1 \mathrm{~s}$, $f_{0}=15 \mathrm{kHz}$.

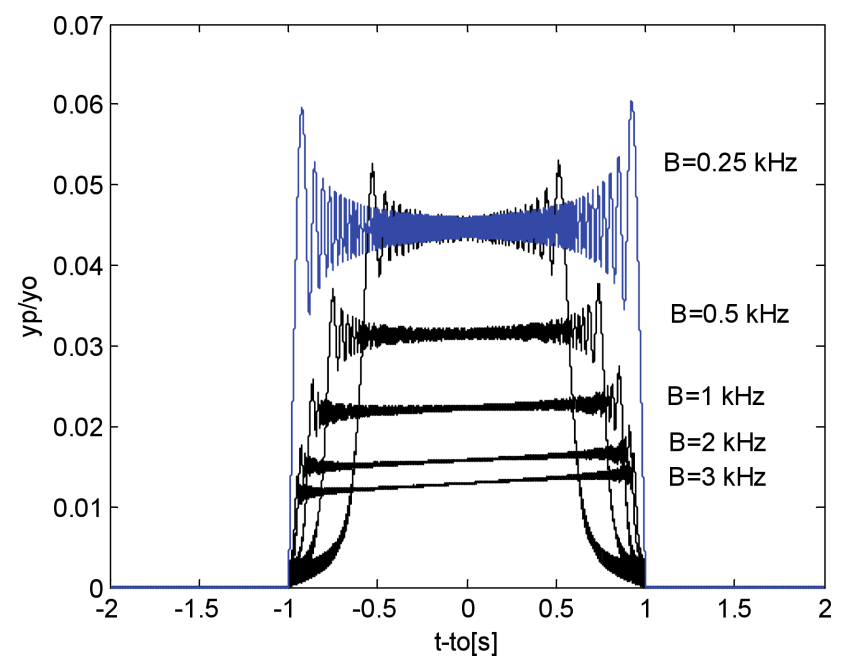

Fig. 15. HFM crosstalk signals at HFM filter output, blue line $v=0 \mathrm{~m} / \mathrm{s}$, black line, $v=10 \mathrm{~m} / \mathrm{s}, T=1 \mathrm{~s}$, $f_{0}=15 \mathrm{kHz}$.

signals for HFM modulation. In both cases, the consequences of the Doppler effect are barely present compared to the effects of the change in modulation direction. The computations have shown that the Doppler effect is also negligible when frequencies and pulse durations change.

\section{Experimental and customer verification}

In order to conduct experimental verification of the numerical computations presented above, crosstalk was measured using the receiver and transmitter of the linear frequency modulated sonar with the central frequency $f_{0}=15.5 \mathrm{kHz}$, a bandwidth of $B=3 \mathrm{kHz}$ and a pulse duration of $T=125 \mathrm{~ms}$ and $50 \mathrm{~ms}$. The test was conducted on Wdzydze Lake by placing of 
a transmitter approximately $900 \mathrm{~m}$ from the sonar receiver. The transmitter and receiver transducers were submerged at a depth of $2 \mathrm{~m}$, and the average depth along the pulse propagation route was app. $20 \mathrm{~m}$.

After passing through the receiver's analogue systems, pulses received by the receiving transducer were sampled with the sampling frequency $f_{s}=4 f_{0}$ and then transformed into digital sequences in a 10 bit analogue to the digital converter. To obtain the quadrature sampling results, pairs of neighbouring samples were recorded by computer memory for every three periods of carrier waves. These were treated as being the real and imaginary parts of the complex sample. A sequence of complex samples forms the lowpass complex envelope of the signal received. Figure 16 shows a module of the complex envelope for an exemplary received signal. The same figure also shows a module of the complex envelope of the signal being transmitted. Figure 17 displays the mod-

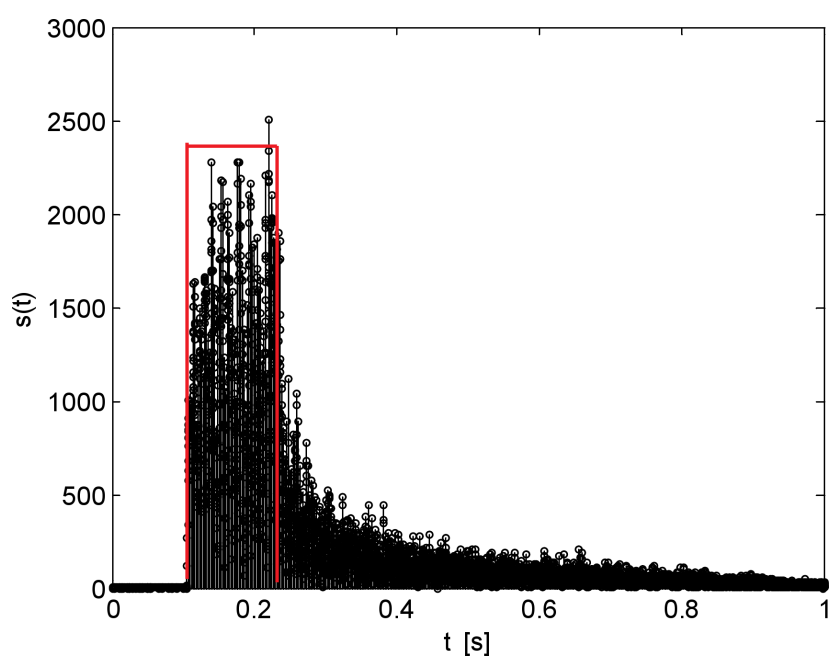

Fig. 16. Complex envelope of received (black) and transmitted (red) signal, $T=250 \mathrm{~ms}$.

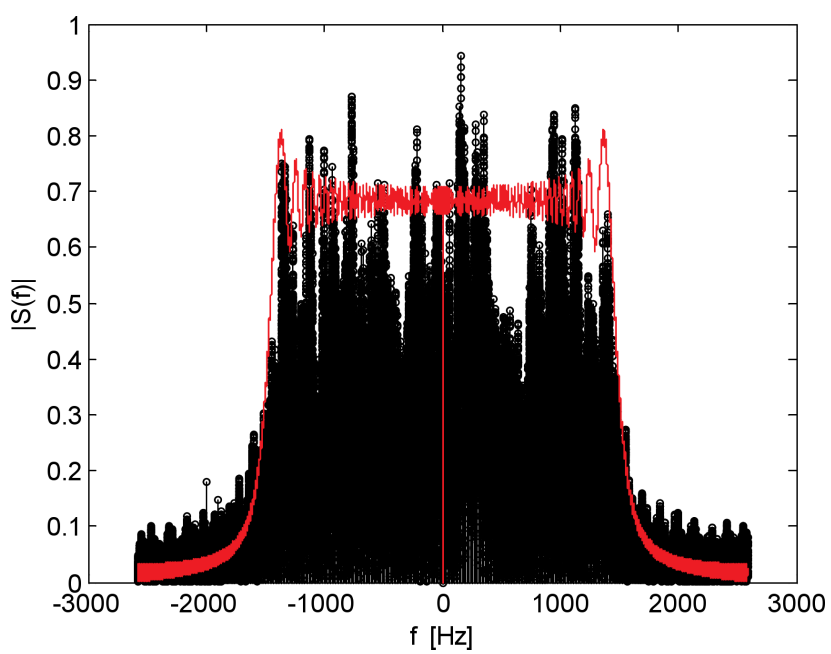

Fig. 17. Module of envelope spectrum of signal received (black) and transmitted (red). ule of the signal spectrum from Fig. 16 and the module of the spectrum from the transmitted signal envelope.

As can be seen in Fig. 16, the received signal is significantly deformed in comparison to the transmitted signal. This is caused by significant reverberations in shallow water. As a consequence of this, the spectrum of the received signal is also deformed, as in Fig. 17.

The complex envelope of the received signal underwent filtration that matched the complex envelope of a transmitted signal with both the same, and opposite, directions of frequency change. The result of this were two functions of correlation between these signals. Figure 18 shows examples of such functions. The mean amplitude of a crosstalk signal with an opposite direction of frequency change was measured, and amounted to $-21.5 \mathrm{~dB}$ (at the worst case $-13.5 \mathrm{~dB}$ ), compared to the filtration when modulation direction was the same. Tables 1 and 2 show the expected theoretical crosstalk value for analogous parameters at $-29.2 \mathrm{~dB}$. The obtained results are worse than the theoretical results, possibly due to a distorted sounding signal resulting from multipath propagation and significant reverberation in shallow water.

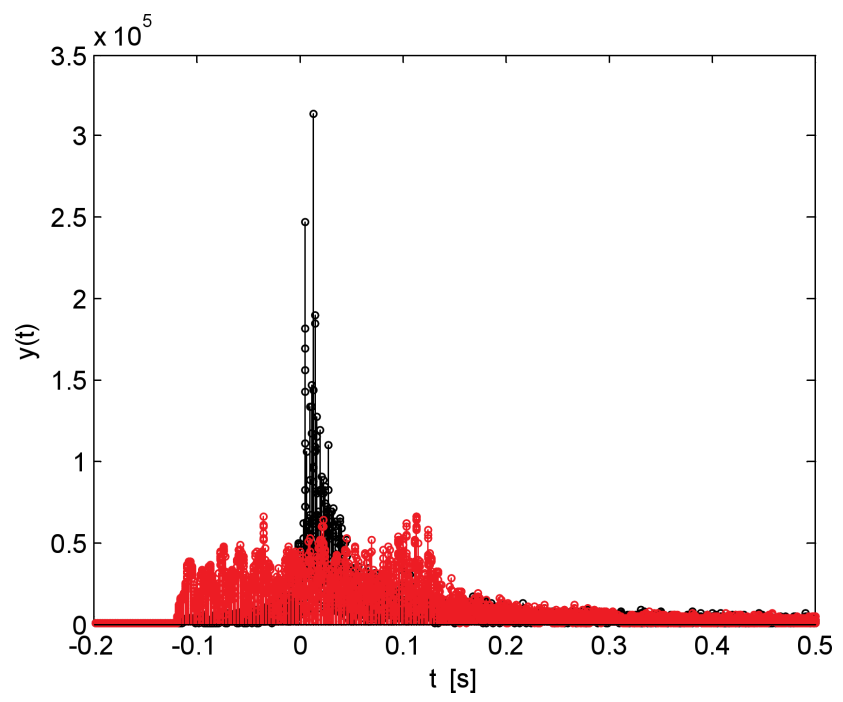

Fig. 18. Complex envelope of signal received after matched filtration with the same (black) and opposite (red) direction of modulation, $T=250 \mathrm{~ms}$.

The above methods for mutual clutter suppression were applied in a Polish Navy helicopter's ASW sonar systems. The users were fully satisfied with the results owing to improved detection and submarine tracking. Mutual clutter suppression was particularly effective when opposite directions of modulation were applied by a pair of cooperating helicopters. An important feature in this is that suppression is sufficient for the unlimited combinations of other settings whose sonar operators freely change depending 
on the specific needs of the search tactic being employed.

\section{References}

1. Elminowicz A., ZajĄCZKowski L. (2007), Mutual interferences reduction between sonars or barrier modules operating in the same area, Hydroacoustics, 10, 39-44.

2. Jedel A., Salamon R., Marszal J., Kaczmarek J., Ostrowski Z. (2001), Mutual interference reduction in pulse sonars with frequency modulation [in Polish], Prace Naukowe Instytutu Telekomunikacji i Akustyki Politechniki Wrocławskiej - Konferencje - XLVIII OSA, 83, 27, 281-284.

3. Marszal J., Salamon R. (2012), Distance Measurement Errors in Silent FM-CW Sonar with Matched Filtering, Metrology and Measurement Systems, XIX, 2, 321-332.

4. Marszal J. (2014), Experimental Investigation of Silent Sonar, Archives of Acoustics, 39, 1, 103-115.
5. Marszal J., Salamon R. (2014), Detection Range of Intercept Sonar for CWFM Signals, Archives of Acoustics, 39, 2, 215-230.

6. Meller M. (2014), Fast clutter cancellation for noise radars via waveform design, Aerospace and Electronic Systems, IEEE Transactions On Aerospace and Electronic Systems, 50, 3, 2327-2333.

7. PJACHEV V. (2008), Sonar signal waveform impact on interference resistance, 16th IEEE European Signal Processing Conference Lausanne 2008, 1-5.

8. RICKS R. (1994), Waveforms for reducing direct blast effects and mutual interference, Technical Report 1676, Naval Command Control and Ocean Surveillance Center San Diego.

9. Salamon R. (2006), Sonar systems [in Polish], Gdańskie Towarzystwo Naukowe, Gdańsk.

10. YANG J., SARKAR T.K. (2006), Doppler-invariant property of hyperbolic frequency modulated waveforms, Microwave and Optical Technology Letters, 48, 6, 1174-1179. 